\title{
A domain decomposition preconditioner of Neumann-Neumann type for the Stokes equations
}

\author{
V. Dolean ${ }^{1}$, F. Nataf ${ }^{2}$ and G. Rapin ${ }^{3}$ \\ 1 Univ. de Nice Sophia-Antipolis, Laboratoire J.-A. Dieudonné, Nice, France. \\ dolean@math.unice.fr \\ ${ }^{2}$ Laboratoire J. L. Lions, Université Pierre et Marie Curie, 75252 Paris Cedex 05, \\ France, nataf@ann.jussieu.fr \\ 3 Math. Dep., NAM, University of Göttingen, D-37083, Germany, \\ grapin@math.uni-goettingen.de
}

Summary. In this paper we recall a new domain decomposition method for the Stokes problem obtained via the Smith factorization. From the theoretical point of view, this domain decomposition method is optimal in the sense that it converges in two iterations for a decomposition into two equal domains. Previous results illustrated the fast convergence of the proposed algorithm in some cases. Our algorithm has shown a more robust behavior than NeumannNeumann or FETI type methods for particular decompositions; as far as general decompositions are concerned, the performances of the three algorithms are similar. Nevertheless, the computations of the singular values of the interface preconditioned problem have shown that one needs a coarse space whose dimension is less than the one needed for the Neumann-Neumann algorithm. In this work we present a new strategy in order to improve the convergence of the new algorithm in the presence of cross points.

\section{Introduction}

The last decade has shown that Neumann-Neumann type algorithms, FETI, and BDDC methods are very efficient domain decomposition methods. Most of the early theoretical and numerical work has been carried out for scalar symmetric positive definite second order problems, see for example $[17,10$, $11,5]$. Then, the method was extended to different other problems, like the advection-diffusion equations [6, 1], plate and shell problems [20] or the Stokes equations $[16,19]$. In the literature one can also find other preconditioners for the Schur complement of the Stokes equations (cf. [19, 2]). Moreover, there exist some Schwarz-type algorithms for non-overlapping decompositions (cf. 
$[15,14,13,18])$. Also FETI [8] and BDDC methods [9] are applied to the Stokes problem with success.

Our work is motivated by the fact that in some sense the domain decomposition methods for Stokes are less optimal than the domain decomposition methods for scalar problems. Indeed, in the case of two subdomains consisting of the two half planes it is well known that the Neumann-Neumann preconditioner is an exact preconditioner for the Schur complement equation for scalar equations like the Laplace problem (cf. [17]). A preconditioner is called exact, if the preconditioned operator simplifies to the identity. Unfortunately, this does not hold in the vector case. It is shown in [4] that the standard Neumann-Neumann preconditioner for the Stokes equations does not possess this property and the construction of an optimal method is explained. Thus, one can expect a very fast convergence for such an algorithm. Indeed, numerical results clearly support our approach. For an application to the compressible Euler equations see [3].

In Section 2 we recall the domain decomposition method for the Stokes system. Section 3 is dedicated to numerical results for the two-dimensional Stokes problem.

\section{DDM for the Stokes equations}

\subsection{Stokes equations}

We consider the stationary Stokes problem in a bounded domain $\Omega \subset \mathbb{R}^{d}$, $d=2,3$. The Stokes equations are given by a velocity $\boldsymbol{u}$ and a pressure $p$ satisfying

$$
\begin{aligned}
-\nu \Delta \boldsymbol{u}+\nabla p+c \boldsymbol{u} & =\boldsymbol{f} \quad \text { in } \Omega, \\
\nabla \cdot \boldsymbol{u} & =0 \quad \text { in } \Omega,
\end{aligned}
$$

and some boundary conditions on $\partial \Omega$. The Stokes problem is a simple model for incompressible flows. The right hand side $\boldsymbol{f}=\left(f_{1}, \ldots, f_{d}\right)^{T} \in\left[L^{2}(\Omega)\right]^{d}$ is a source term, $\nu$ is the viscosity and $c \geq 0$ is a constant reaction coefficient. Very often $c$ stems from an implicit time discretization and then $c$ is given by the inverse of the time step size. In the following we denote the $d$-dimensional Stokes operator by $\mathcal{S}_{d}(\boldsymbol{u}, p):=(-\nu \Delta \boldsymbol{u}+c \boldsymbol{u}+\nabla p, \nabla \cdot \boldsymbol{u})$. In the following we will restrict to the two-dimensional case $(d=2)$ but the three-dimensional formulation can be found in [4].

\subsection{A new algorithm for the Stokes equations}

We further introduce the stress depending on a velocity $\boldsymbol{u}=(u, v)$, a pressure $p$ and the unit normal vector $\boldsymbol{n}$ on the boundary: 


$$
\boldsymbol{\sigma}_{\boldsymbol{n}}(\boldsymbol{u}, p):=\nu \frac{\partial \boldsymbol{u}}{\partial \boldsymbol{n}}-p \boldsymbol{n}
$$

For any vector $\boldsymbol{u}$, its normal (resp. tangential) component on the interface is $u_{\boldsymbol{n}_{i}}=\boldsymbol{u} \cdot \boldsymbol{n}_{\boldsymbol{i}}\left(\operatorname{resp} . u_{\boldsymbol{\tau}_{i}}=\boldsymbol{u} \cdot \boldsymbol{\tau}_{i}\right)$. We denote $\sigma_{\boldsymbol{n}_{i}}^{i}\left(\boldsymbol{u}_{i}, p_{i}\right):=\boldsymbol{\sigma}_{\boldsymbol{n}_{i}}\left(\boldsymbol{u}_{i}, p_{i}\right) \cdot \boldsymbol{n}_{i}$ and $\sigma_{\boldsymbol{\tau}_{i}}^{i}\left(\boldsymbol{u}_{i}, p_{i}\right):=\boldsymbol{\sigma}_{\boldsymbol{n}_{i}}\left(\boldsymbol{u}_{i}, p_{i}\right) \cdot \boldsymbol{\tau}_{i}$ as the normal and tangential parts of $\boldsymbol{\sigma}_{\boldsymbol{n}_{i}}$, respectively. We now present the new algorithm for the Stokes equations for a general decomposition into non overlapping subdomains $\bar{\Omega}=\cup_{i=1}^{N} \bar{\Omega}_{i}$. We denote by $\Gamma_{i j}$ the interface between subdomains $\Omega_{i}$ and $\Omega_{j}, i \neq j$. The new algorithm for the Stokes system is:

ALGORITHM 1 Starting with an initial guess $\left(\left(\boldsymbol{u}_{i}^{0}, p_{i}^{0}\right)\right)_{i=0}^{N}$ satisfying $\boldsymbol{u}_{i, \boldsymbol{\tau}_{i}}^{0}=$ $\boldsymbol{u}_{j, \boldsymbol{\tau}_{j}}^{0}$ and $\sigma_{\boldsymbol{n}_{i}}^{i}\left(\boldsymbol{u}_{i}^{0}, p_{i}^{0}\right)=\sigma_{\boldsymbol{n}_{j}}^{j}\left(\boldsymbol{u}_{j}^{0}, p_{j}^{0}\right)$ on $\Gamma_{i j}, \forall i, j, i \neq j$, the correction step $i s$ expressed as follows for $1 \leq i \leq N$ :

$$
\begin{cases}\mathcal{S}_{2}\left(\tilde{\boldsymbol{u}}_{i}^{n+1}, \tilde{p}_{i}^{n+1}\right) & =0 \quad \text { in } \Omega_{i} \\ \tilde{u}_{i, \boldsymbol{n}_{i}}^{n+1} & =-\frac{1}{2}\left(u_{i, \boldsymbol{n}_{i}}^{n}+u_{j, \boldsymbol{n}_{j}}^{n}\right) \quad \text { on } \Gamma_{i j} \\ \sigma_{\boldsymbol{\tau}_{i}}^{i}\left(\tilde{\boldsymbol{u}}_{i}^{n+1}, \tilde{p}_{i}^{n+1}\right) & =-\frac{1}{2}\left(\sigma_{\boldsymbol{\tau}_{i}}^{i}\left(\boldsymbol{u}_{i}^{n}, \tilde{p}_{i}^{n}\right)+\sigma_{\boldsymbol{\tau}_{j}}^{j}\left(\boldsymbol{u}_{j}^{n}, \tilde{p}_{j}^{n}\right)\right) \quad \text { on } \Gamma_{i j},\end{cases}
$$

followed by an updating step for $1 \leq i \leq N$ :

$$
\begin{cases}\mathcal{S}_{2}\left(\boldsymbol{u}_{i}^{n+1}, p_{i}^{n+1}\right) & =\boldsymbol{g} \quad \text { in } \Omega_{i} \\ \boldsymbol{u}_{i, \boldsymbol{\tau}_{i}}^{n+1} & =\boldsymbol{u}_{i, \boldsymbol{\tau}_{i}}^{n}+\frac{1}{2}\left(\tilde{\boldsymbol{u}}_{i, \boldsymbol{\tau}_{i}}^{n+1}+\tilde{\boldsymbol{u}}_{j, \boldsymbol{\tau}_{j}}^{n+1}\right) \quad \text { on } \Gamma_{i j} \\ \sigma_{\boldsymbol{n}_{i}}^{i}\left(\boldsymbol{u}_{i}^{n+1}, p_{i}^{n+1}\right) & =\sigma_{\boldsymbol{n}_{i}}^{i}\left(\boldsymbol{u}_{i}^{n}, p_{i}^{n}\right) \\ & +\frac{1}{2}\left(\sigma_{\boldsymbol{n}_{i}}^{i}\left(\tilde{\boldsymbol{u}}_{i}^{n+1}, \tilde{p}_{i}^{n+1}\right)+\sigma_{\boldsymbol{n}_{j}}^{j}\left(\tilde{\boldsymbol{u}}_{j}^{n+1}, \tilde{p}_{j}^{n+1}\right)\right) \text { on } \Gamma_{i j} .\end{cases}
$$

We have

Proposition 1. For a domain $\Omega=\mathbb{R}^{2}$ divided into two non overlapping half planes, Algorithm 1 converges in two iterations.

The new algorithm for the Stokes system is reminiscent of the hybrid approach presented in [7]. Indeed, in both cases, the interface conditions are mixed Dirichlet and Neumann type boundary conditions. However, our approach is different in the sense that it shows the good combination of stress and displacement for the interface conditions in both $2 \mathrm{~d}$ and $3 \mathrm{~d}$ (see [4] for details).

\section{Numerical results}

In this section we will analyze the performance of the new algorithm in the two-dimensional case. As in [4], we consider the domain $\Omega=(0.2,1.2) \times$ $(0.1,1.1)$ decomposed into $N \times N$ subdomains (in the presence of cross points). We choose the right hand side $\boldsymbol{f}$ such that the exact solution is given by $u(x, y)=\sin (\pi x)^{3} \sin (\pi y)^{2} \cos (\pi y), v(x, y)=-\sin (\pi x)^{2} \sin (\pi y)^{3} \cos (\pi x)$ and 
$p(x, y)=x^{2}+y^{2}$. The viscosity $\nu$ is always 1 . The interface system is solved by GMRES. In all tables we count the number of iterations needed to reduce the $L^{\infty}$ norm of the error by the factor $T O L=10^{-6}$ :

$$
\max _{i=1, \ldots, N}\left\|U_{k}^{i}-U_{h}\right\|_{L^{\infty}\left(\Omega_{i}\right)} \leq 10^{-6},
$$

where $U_{k}^{i}=\left(u_{k}, v_{k}, p_{k}\right)^{i}$ is the discrete solution of iteration step $k$ in subdomain $\Omega_{i}$ and $U_{h}=\left(u_{h}, v_{h}, p_{h}\right)$ is the global discrete solution computed by a direct solver applied to the global problem.

A problem in Algorithm 1 is that in the correction step, the local matrices may be singular (the local problems are ill-posed for the pressure, the latter being defined up to an additive constant). To overcome this difficulty we chose to add a penalization term $\varepsilon p$ with $\varepsilon$ sufficiently small to the divergence equation. This penalization term leads however still to ill-conditioned local matrices and an ill-conditioned interface problem. Thus, the reduction of the Euclidean norm of the residual is not a good indicator for the convergence of the algorithm as can be seen in Figure 3:
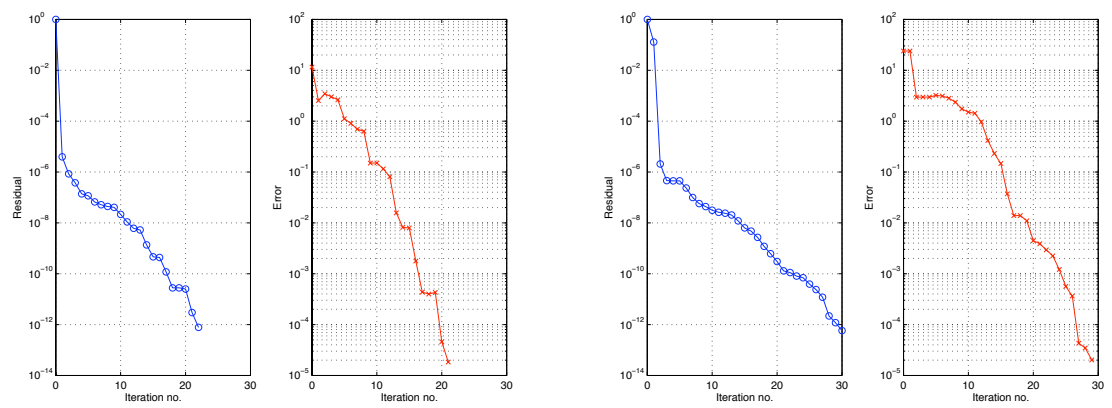

Fig. 1. Convergence of the GMRES algorithm (residual and error) for $3 \times 3$ (left) and $4 \times 4$ (right) decompositions for $\varepsilon>0$

This is also due to the presence of the large eigenvalues in the spectrum, as seen in the Table 1.

A very simple way to eliminate the large eigenvalues is to avoid using the penalization term: the local problems are now singular. Consider a local matrix $A$ which corresponds to interior subdomains in the correction (preconditioning) step. It will be singular of co-rank 1 . The null space is formed by a vector whose components are constant non-zero only for the pressure components. It can be easily shown that the matrix $B+f \cdot e^{t}$ is invertible if we choose $e$ (resp. $f$ ) to be a vector non-orthogonal to $\operatorname{ker}(A)\left(\right.$ resp. $\left.\operatorname{ker}\left(A^{T}\right)\right)$. In our case it is sufficient to take (in order to preserve the sparsity of the matrix $A)$ a vector with null components except for one non-zero component chosen 


\begin{tabular}{|c|c|c|c|}
\hline$N \times N$ & No. of large eigenvalues & $N \times N$ & No. of large eigenvalues \\
\hline $2 \times 2$ & 0 & $6 \times 6$ & 16 \\
$3 \times 3$ & 1 & $7 \times 7$ & 25 \\
$4 \times 4$ & 4 & $8 \times 8$ & 36 \\
$5 \times 5$ & 9 & $9 \times 9$ & 49 \\
\hline
\end{tabular}

Table 1. Number of eigenvalues which are larger than 10 in modulus for a $N \times N$ decomposition.

in the right position. Afterward, for any right hand side $b$ in the $\operatorname{Im}(A)$, the solution of $B x=b$ verifies $A x=b$. In this case no more large eigenvalues will be present in the spectrum and the convergence of the residual will reflect more accurately the convergence of the error as one can see in Figure 3.
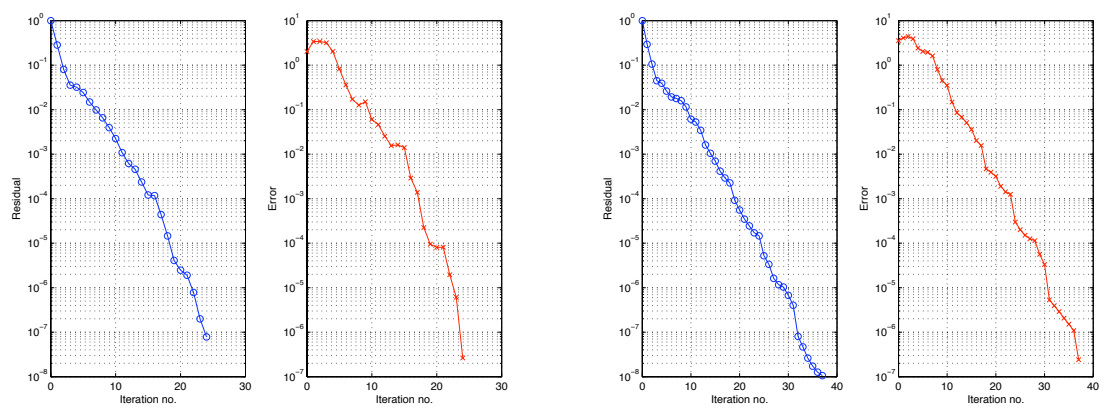

Fig. 2. Convergence of the GMRES algorithm (residual and error) for $3 \times 3$ (left) and $4 \times 4$ (right) decompositions for $\varepsilon=0$

Nevertheless the convergence is still very sensitive to the number of subdomains, which shows the necessity of introducing a coarse space correction in the algorithm. This bad convergence is mainly due to the presence of small eigenvalues in the spectrum of the interface operator (see Figure 3).

We need to eliminate the small eigenvalues which can cause bad convergence. In order to do this we will first notice that the error during the iterations of the GMRES method is mainly localized in the corners, as seen in Figure 4 where the error on component $p$ is visualized.

A solution to this problem could be a deflation method applied to the preconditioning step as seen in [12], where the deflated vectors contain constant non-zero elements only for the corner components of the solution. As a result, we obtain a better convergence than before. It is however not optimal, since it is dependent on the number of subdomains (see Figure 3). 

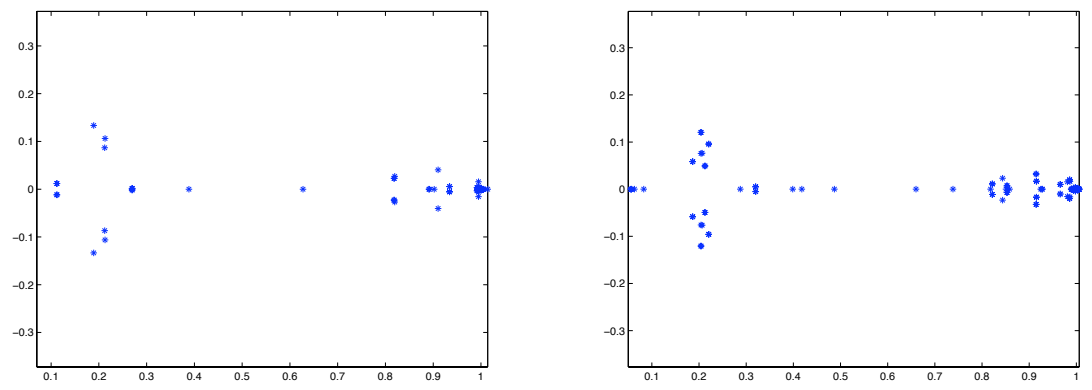

Fig. 3. Eigenvalues of the interface preconditioned operator for $3 \times 3$ (left) and $4 \times 4$ (right) decompositions.

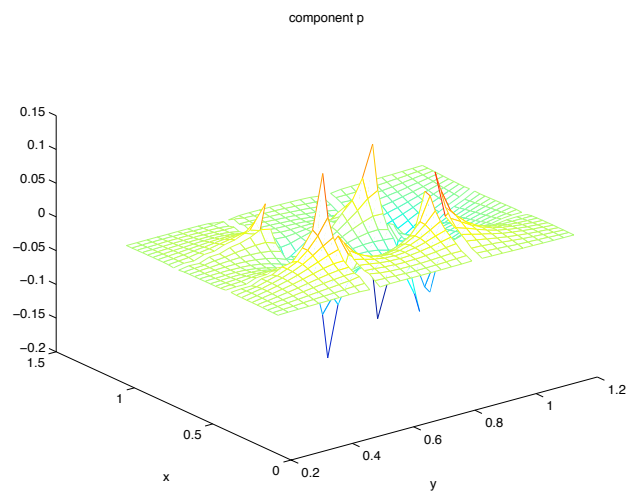

Fig. 4. Error on the component $p$ of the solution

By looking at the spectrum (Figure 3), we can see that there are still small eigenvalues that have not been taken care of by the deflation method.

As a conclusion we can state that even if the strategy presented is not yet optimal, it leads to an improvement of the previous algorithm (since it eliminates a part of small eigenvalues) and could pave the way to the construction of a more scalable method.

\section{References}

[1] Y. Achdou, P. Le Tallec, F. Nataf, and M. Vidrascu. A domain decomposition preconditioner for an advection-diffusion problem. Comput. Methods Appl. Mech. Engrg., 184:145-170, 2000. 

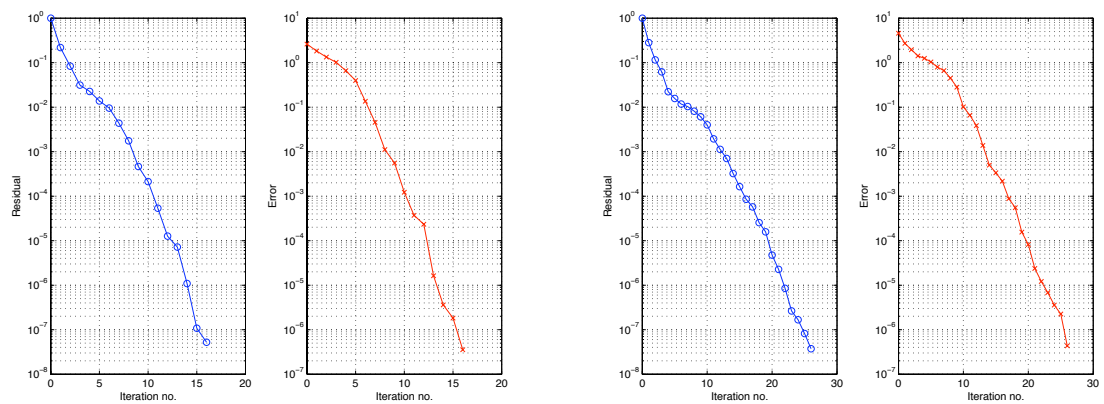

Fig. 5. Convergence of the deflated GMRES algorithm (residual and error) for $3 \times 3$ (left) and $4 \times 4$ (right) decompositions.
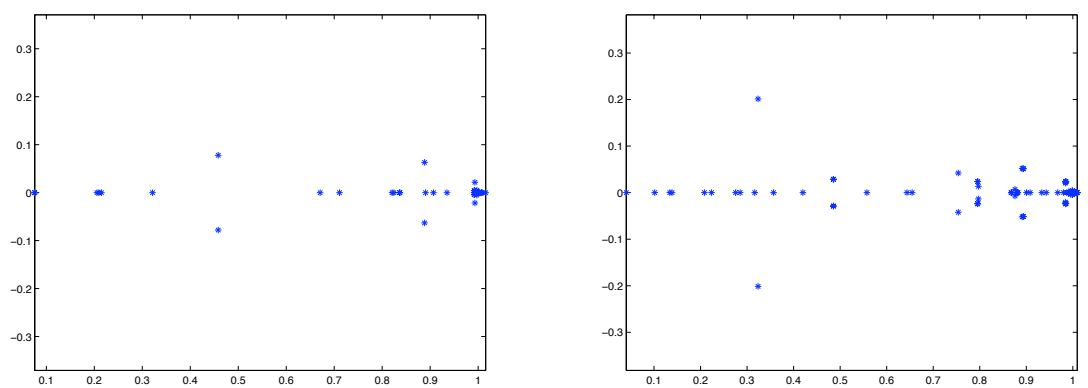

Fig. 6. Eigenvalues of the interface preconditioned operator for $3 \times 3$ (left) and $4 \times 4$ (right) decompositions.

[2] M. Ainsworth and S. Sherwin. Domain decomposition preconditioners for $\mathrm{p}$ and hp finite element approximations of Stokes equations. Comput. Methods Appl. Mech. Engrg., 175:243-266, 1999.

[3] V. Dolean and F. Nataf. A New Domain Decomposition Method for the Compressible Euler Equations. ESAIM-M2AN(Modelisation Mathematique et Analyse Numerique), 40(4):689-703, 2006.

[4] V. Dolean, F. Nataf, and G. Rapin. Deriving a new domain decomposition method for the Stokes equations using the Smith factorization. Math. Comp., 78:789-814, 2009.

[5] Ch. Farhat and F.-X. Roux. A Method of Finite Element Tearing and Interconnecting and its Parallel Solution Algorithm. Internat. J. Numer. Methods Engrg., 32:1205-1227, 1991.

[6] L. Gerardo-Giorda, P. Le Tallec, and F. Nataf. A Robin-Robin preconditioner for advection-diffusion equations with discontinuous coefficients. 
Comput. Methods Appl. Mech. Engrg., 193:745-764, 2004.

[7] P. Gosselet and C. Rey. Non-overlapping domain decomposition methods in structural mechanics. Arch. Comput. Methods Engrg., 13(4):515-572, 2006.

[8] J. Li. A Dual-Primal FETI method for incompressible Stokes equations. Numer. Math., 102:257-275, 2005.

[9] J. Li and O. Widlund. BDDC algorithms for incompressible Stokes equations. SIAM J. of Numer. Anal., 44(6):2432-2455, 2006.

[10] J. Mandel. Balancing domain decomposition. Comm. on Applied Numerical Methods, 9:233-241, 1992.

[11] J. Mandel and M. Brezina. Balancing domain decomposition: Theory and performance in two and three dimensions. UCD/CCM report 2, 1993.

[12] R. Nabben and C. Vuik. A comparison of abstract versions of deflation, balancing and additive coarse grid correction preconditioners. Numer. Linear Algebra Appl., 15:355-372, 2008.

[13] F. Nataf. Interface Conditions for Domain Decomposition Methods for 2D and 3D Oseen equations. C. R. Acad. Sci., Paris, Ser. I 324:11551160, 1997.

[14] F.-C. Otto and G. Lube. A nonoverlapping domain decomposition method for the Oseen equations. Math. Models Methods Appl. Sci., 8:1091-1117, 1998.

[15] F.-C. Otto, G. Lube, and L Müller. An iterative substructuring method for div-stable finite element approximations of the Oseen problem. Computing, 67:91-117, 2001.

[16] L.F. Pavarino and O.B. Widlund. Balancing Neumann-Neumann methods for incompressible Stokes equations. Comm. Pure Appl. Math., 55:302-335, 2002.

[17] Y.H. De Roeck and P. Le Tallec. Analysis and Test of a Local Domain Decomposition Preconditioner. In R. Glowinski and Y.A. Kuznetsov and G. Meurant and J. Periaux and O.B. Widlund editors, Fourth International Symposium on Domain Decomposition Methods for Partial Differential Equations, 1991.

[18] E. Ronquist. A Domain Decomposition Solver for the Steady NavierStokes Equations. In A. Ilin and L. Scott, editors, Proc. of ICOSAHOM.95, pages 469-485. Houston Journal of Mathmatics, 1996.

[19] P . Le Tallec and A. Patra. Non-overlapping domain decomposition methods for adaptive hp approximations of the Stokes problem with discontinuous pressure fields. Comput. Methods Appl. Mech. Engrg., 145:361-379, 1997.

[20] P. Le Tallec, J. Mandel, and M. Vidrascu. A Neumann-Neumann Domain Decomposition Algorithm for Solving Plate and Shell Problems. SIAM J. Numer. Anal., 35:836-867, 1998. 